\title{
Estrategias de afrontamiento: factores de protección en el consumo de alcohol, tabaco y cannabis.
}

\author{
Noelia llorens Aleixandre*, Miguel Perelló del Río**, Alfonso Palmer Pol*** \\ * Profesor ayudante del Área de Metodología, Universidad de las Islas Baleares. \\ ** Psicólogo práctica privada. Centro de Aplicaciones Psicológicas. Valencia. \\ ** Profesor Titular del Área de Metodología, Universidad de las Islas Baleares.
}

\section{RESUMEN}

Objetivo: El objetivo del presente estudio es analizar el valor explicativo de las estrategias de afrontamiento, habilidades sociales y habilidades propias, en el consumo de diferentes sustancias adictivas legales e ilegales.

Método: Se administró un cuestionario anónimo, a una muestra de 314 jóvenes adultos, entre 18 y 30 años, desarrollado para medir la cantidad de sustancia consumida en una semana, las estrategias de afrontamiento, habilidades sociales y habilidades propias. Las variables de respuesta analizadas fueron el número de bebidas destiladas (whisky, vodka, etc..), número de bebidas fermentadas (cerveza, vino, etc...) y número de cigarrillos de cannabis y de tabaco.

Resultados: Déficits en estrategias de afrontamiento como "Pensar en las consecuencias negativas" disminuye el consumo de bebidas fermentadas y de cannabis en un $24 \%$ y en un $40 \%$ respectivamente. La habilidad propia "Ser disciplinado" disminuye el consumo de bebidas destiladas, fermentadas y cannabis, en un $38 \%, 31 \%$ y $33 \%$ respectivamente. Déficits en habilidades sociales, como la dificultad para relacionarse con personas del sexo opuesto, influyen disminuyendo el consumo de bebidas destiladas (45\%), de cannabis $(70 \%)$ y aumentando el consumo de tabaco por cuatro.

Conclusiones: Únicamente déficits en habilidades sociales influyen en el consumo de tabaco. Déficits en habilidades sociales y propias influyen en el consumo que los sujetos hacen de las bebidas destiladas y de cannabis y déficits en estrategias de afrontamiento son las que llevarán a un sujeto a consumir bebidas fermentadas. La modificación de todos éstos déficits puede permitir hacer programas de prevención más efectivos.

Palabras clave: Estrategias Afrontamiento, Poisson, Sobredispersión, Cannabis, Tabaco, Alcohol.

\section{ABSTRACT}

Purpose: The aim of this study is to analyse the explanatory value of coping skills, own skills and social skills, over use of different legal and illegal addictive substances in youth adults' population.

Methods: An anonymous questionnaire was administered to a sample 314 youths adults, between 18 and 30 years. The instrument measures the quantity of substance consumed in one week, coping skills, social skills and own skills. The analysed answer variables were the number of consumed distilled drinks (whisky, vodka, etc.), fermented drinks (beer, wine, etc.) and cannabis cigarettes and tobacco.

Results: Lacks in coping skills like to "Think of the negative consequences" diminishes the use of fermented drinks and cannabis a $24 \%$ and $40 \%$ respectively. The own skill "To be disciplined" diminishes the use of distilled drinks, fermented drinks and cannabis a 38\%, 31\% and 33\% respectively. Lacks in social skills, as the difficulty to be related with people of the opposite sex, influences diminishing the use of distilled drinks $(45 \%)$ and cannabis (70\%), and increasing the use of tobacco fourth time.

Conclusions: Lacks in social skills are the only significant influence in the use of tobacco. Lacks in social and own abilities have an effect to the use of distilled drinks and cannabis, and lacks in coping skills facilitate a subject to use fermented drinks. The modification of all these lacks could guide to develop effective prevention programs.

Key words: Coping Skills, Poisson, Overdispersion, Cannabis, Tobacco, Alcohol. 


\section{INTRODUCCIÓN}

E n el pasado uno de los primeros acercamientos teóricos a las conductas adictivas, fue el modelo de enfermedad, que enfatizaba la importancia de los parámetros biológicos de las adicciones haciendo hincapié en los efectos farmacológicos de las sustancias. Esto implicaba que la persona adicta no tenía control voluntario sobre su conducta adictiva, ya que dicha conducta estaba determinada por factores fisiológicos internos como compulsiones, impulsos o deseos irresistibles a consumir (Marlatt, 1985). Una alternativa al modelo de enfermedad ha sido la Teoría del Aprendizaje Social de Bandura (1977) que explica la conducta humana como un fenómeno de aprendizaje basándose en las leyes del condicionamiento clásico, operante y vicario. Este modelo y los derivados del mismo, enfatizan la capacidad de autocontrol y/o autorregulación que el sujeto tiene sobre su conducta, derivándose tratamientos que implican el entrenamiento en estrategias de afrontamiento.

Dentro de las teorías cognitivo-conductuales sobre los procesos adictivos, el Modelo de Afrontamiento al Estrés (Wills y Shiffman, 1985; Wills y Hirky, 1996) asume que el consumo de sustancias podría ser una respuesta del sujeto a los estresores vitales a los que se enfrenta a lo largo de su vida, el consumo de sustancias reduciría los efectos negativos del estrés o aumentaría la capacidad del sujeto para hacer frente a los mismos. Siguiendo este modelo se puede deducir que si el sujeto tiene habilidades adecuadas y adaptativas para afrontar el estrés tendrá menos tendencia a desarrollar trastornos adictivos que si carece de estas habilidades.

Wagner, Myers y Ininch, (1999) en un estudio para validar empíricamente el modelo de afrontamiento al estrés, comprobaron que los adolescentes que utilizan estrategias centradas en el afrontamiento a las situaciones de estrés consumían menos que los que utilizaban estrategias de evitación, similares resultados se han encontrado en población adulta (Finney y Moos, 1995). Estos estudios apoyan la idea de que las estrategias de afrontamiento al estrés predicen el uso de drogas en adolescentes y adultos. Por otro lado, el modelo de Prevención de Recaídas de Marlatt y Gordon (1985) hace énfasis en la importancia de las estrategias de afrontamiento en el uso de drogas. Este modelo señala que después de un periodo de abstinencia se puede producir la vuelta al consumo cuando el sujeto tiene que enfrentarse a situaciones de alto riesgo y no utiliza las estrategias de afrontamiento adecuadas (Marlatt y Gordon, 1980). Este modelo subraya la importancia del entrenamiento en estrategias de afrontamiento tanto conductuales como cognitivas (Chaney, O'Leary y Marlatt, 1978). Estas estrategias de afrontamiento consisten básicamente en prever las posibles situaciones de alto riesgo futuras, el entrenamiento en resolución de problemas (D'Zurilla y Goldfried, 1971), en dar respuestas asertivas en dichas situaciones (Flowers, 1975) y en estrategias de manejo del estrés (Lazarus y Folkman, 1984). Por su parte el Modelo Transteórico del Cambio (Prochaska y DiClemente, 1982; Prochaska, DiClemente y Norcross, 1992; Prochaska y Prochaska, 1993) describe y delimita las variables y elementos que los sujetos ponen en práctica de forma intencional en el proceso de cambio de una conducta adictiva. Propone que en el proceso de cambio de una conducta adictiva se dan tres dimensiones: estadios, procesos y niveles de cambio. Los estadios hacen referencia a diferentes etapas motivacionales, los procesos son conductas observables o no que los sujetos realizan encaminadas a modificar su comportamiento adictivo y los niveles son cambios necesarios que se tienen que producir para abandonar una conducta adictiva. Estos autores cuando hablan de procesos se están refiriendo a las diferentes estrategias de afrontamiento que tienen que poner en práctica los sujetos en los diferentes estadios de cambio. Hay diferentes estudios que demuestran la influencia de las habilidades sociales en el consumo de drogas. Se ha detectado que los adolescentes que utilizan más habilidades asertivas (decir "no") y habilidades referidas a toma de decisiones consumen menos drogas ilegales (Barkin, Smith y DuRant, 2002). Hay estudios que apoyan el entrenamiento de habilidades sociales para el tratamiento de diferentes adicciones (Chaney y otros, 1978, Chaney, Roszell y Cummings, 1982; Marlatt y Gordon, 1980; Monti, Abrams, Binkoff y Zwick, 1986), por lo que se parte de que las personas que abusan de sustancias tienen déficit de habilidades sociales que pueden ser de dos tipos: Secundarios, que hacen referencia a falta de asertividad en situaciones relacionadas con el consumo, o primarios, problemas de habilidades sociales generales en diversas situaciones (Trower, Yardley, Briant y Shaw, 1978). Todas estas teorías y estudios muestran la importancia de las estrategias de afrontamiento, tanto conductuales como cognitivas, en el consumo de sustancias adictivas.

El objetivo del presente estudio es analizar, en una muestra de jóvenes adultos, el valor explicativo de las estrategias de afrontamiento cognitivo y conductuales, entre ellas las habilidades sociales especificas a la situación de consumo y las generales a situaciones interpersonales. Concretamente se pretende averiguar el peso de cada una de las variables en la explicación de la conducta de consumo de diferentes drogas, como son el consumo de bebidas destiladas, fermentadas, tabaco y cannabis. La variable a explicar es la variable de respuesta número de bebidas, número de cigarrillos y número de cigarrillos de THC consumidos en una semana. Como las variables de respuesta son variables de recuento se aplicará para su análisis, en 
el contexto del Modelo Lineal Generalizado, el modelo de regresión de Poisson.

\section{MÉTODO}

La muestra estaba formada por 314 sujetos de edades comprendidas entre los 18 y los 30 años, con una edad media de 22.7 años. Por sexos, el $53.9 \%$ eran hombres y el $46.1 \%$ eran mujeres. La muestra estaba formada por 291 universitarios y 23 no universitarios. Se utilizó un muestreo incidental llevado a cabo en varios edificios del Campus Universitario correspondientes a diferentes estudios, así como en zonas de marcha de la ciudad.

En cuanto al consumo que presentaban, 22.1\% eran No consumidores (Si no habían probado nunca ninguna de las sustancias), el $12.1 \%$ eran experimentadores o ex-consumidores (habían probado en una o dos ocasiones o habían consumido alguna droga en otro tiempo, pero actualmente no consumían ninguna droga), el $69.4 \%$ eran consumidores ocasionales de alguna de las sustancias y el $55.4 \%$ eran consumidores habituales (fin de semana, entre semana y a diario) de alguna de las sustancias analizadas. Se les administró un cuestionario anónimo desarrollado para medir la frecuencia de uso de diferentes sustancias adictivas, así como las habilidades propias, habilidades personales y estrategias de afrontamiento.

Los cuestionarios fueron administrados por un entrevistador, que preguntaba personalmente a los sujetos de forma individual y recogía las respuestas dadas por estos.

Las variables explicativas introducidas en el modelo fueron: a) Estrategias de afrontamiento, consideradas como habilidades específicas puestas en práctica por el sujeto para evitar el consumo. b) Habilidades propias, recogidas como percepción que tiene el sujeto de sus habilidades generales frente a cualquier situación problemática. c) Habilidades sociales, percepción que tiene el sujeto de sus habilidades de interrelación social (ver tabla1).

Todas estas variables eran variables categóricas binarias para evitar las respuestas de tendencia intermedia y obligar a contestar en algún sentido.

Las variables de respuesta modeladas fueron el número de copas de bebidas destiladas (ginebra, ron, whisky, combinados, etc.) consumidas en una semana, el número de copas de bebidas fermentadas (vino, cerveza, cava, etc.) consumidas en una semana, el número de cigarrillos consumidos en una semana y el número de cigarrillos de THC consumidos en una semana.

Se planteó un diseño de carácter explicativo, el objetivo del cual es comprobar los factores que per-

\section{TABLA 1: Items del cuestionario.}

\begin{tabular}{|lll|}
\hline Estrategias de Afrontamiento & Habilidades Propias & Habilidades Sociales \\
\hline $\begin{array}{l}\text { 1-Decir que no quiero cuando } \\
\text { me ofrecen. }\end{array}$ & $\begin{array}{l}\text { 1-Tiene facilidad para encontrar } \\
\text { soluciones cuando tiene un } \\
\text { problema. }\end{array}$ & $\begin{array}{l}\text { 1-Tienes dificultades para conocer } \\
\text { gente nueva. }\end{array}$ \\
\hline $\begin{array}{l}\text { 2-Evitar lugares y personas } \\
\text { relacionados con drogas. }\end{array}$ & $\begin{array}{l}\text { 2-Se considera una persona } \\
\text { disciplinada. }\end{array}$ & $\begin{array}{l}\text { 2-Tienes dificultades para expresar tus } \\
\text { sentimientos. }\end{array}$ \\
\hline $\begin{array}{l}\text { 3-Pensar en las consecuencias } \\
\text { negativas que acarrea el } \\
\text { consumo. }\end{array}$ & $\begin{array}{l}\text { 3-Sabe distraerse cuando } \\
\text { quiere dejar de pensar } \\
\text { en algo. }\end{array}$ & $\begin{array}{l}\text { 3-Te cuesta iniciar, mantener o cerrar } \\
\text { una conversación con personas que } \\
\text { no conoces. }\end{array}$ \\
\hline 4-Simplemente no llevar dinero. & $\begin{array}{l}\text { 4-Sabe relajarse sin utilizar } \\
\text { drogas. }\end{array}$ & $\begin{array}{l}\text { 4-Tienes problemas para relacionarse } \\
\text { con personas del sexo opuesto. }\end{array}$ \\
\hline & $\begin{array}{l}\text { 5-Tienes dificultades para decir "no" a } \\
\text { otras personas. }\end{array}$ \\
\hline & $\begin{array}{l}\text { 6-Te resulta muy difícil negarte } \\
\text { cuando te ofrecen implicarte en } \\
\text { conductas de consumo de sustancias. }\end{array}$ \\
\hline
\end{tabular}


miten predecir las variables de respuesta número de copas consumidas de bebidas fermentadas y destiladas, número de cigarrillos y número de cigarrillos de THC consumidos en una semana. Las bebidas se han convertido a UBES (Unidades de bebidas estándar) para realizar los análisis.

El modelo estadístico utilizado para analizar los datos se enmarca dentro del Modelo Lineal Generalizado, concretamente se aplicará el modelo de regresión de Poisson como modelo adecuado para datos de recuento. El análisis se realizó con el Stata 8.0.

\section{RESULTADOS}

Se ha utilizado la prueba de hipótesis basada en la regresión (Cameron y Trivedi, 1998) para evaluar el supuesto de equidispersión del modelo de regresión de Poisson, obteniéndose que en bebidas destiladas $(t=2,82 ; p=0.006)$, en tabaco $(t=7,96 ; p<0.001)$ y en cannabis $(t=4,49 ; p<0.001)$ se incumple el supuesto, mientras que éste se cumple en bebidas fermentadas $(t=1,46 ; p=0.148)$, por lo que en los tres primeros casos se ha utilizado el error estándar robusto.

Aplicamos el modelo a las diferentes muestras de consumidores y las variables que aparecen como explicativas para cada una de las variables de respuesta son las que se presentan a continuación.

En bebidas fermentadas encontramos que los sujetos que dicen utilizar la estrategia de afrontamiento "Evito lugares y personas relacionados con drogas" consumen un $46 \%$ menos respecto a los que no utilizan esta estrategia ( $b=-0.617, p=0.003)$, siendo el número esperado de Ubes para los que utilizan esta estrategia de 2.8 Ubes menos a la semana. Por otro lado, los que dicen que usan la estrategia de afrontamiento "No llevar dinero", multiplican por 6 el consumo de estas sustancias $(b=1.81, p=0.001)$, presentando un valor esperado de 29 Ubes a la semana más que los que no utilizan esta estrategia de afrontamiento.

Aquellos que piensan en las consecuencias negativas que acarrea el consumo disminuyen un $24 \%$ su consumo respecto a los que no utilizan esta estrategia de afrontamiento $(b=-0.271, p=0.016)$, lo que conlleva disminuir 1.5 Ubes a la semana su valor esperado en Ubes consumidas.

Las personas que dicen ser disciplinadas consumen un $31 \%$ menos que las que señalan que no son disciplinadas $(b=-0.365, p=0.003)$, esperándose un consumo de 2.5 Ubes menos a la semana. Si hablamos de los que señalan dificultades para negarse cuando les ofrecen implicarse en conductas de consumo de sustancias, vemos como consumen casi el triple, esperando un consumo de 10.2 Ubes más a la semana $(b=1.031, p<0.001)$.

Los que señalan facilidad para solucionar problemas consumen un $31 \%$ más de bebidas fermentadas $(b=0.270, p=0.005), 1.46$ Ubes más a la semana, que los que dicen que no les resulta fácil solucionar problemas.

En bebidas destiladas los sujetos que se consideran disciplinados consumen un $38 \%$ menos que los que señalan no ser disciplinados, concretamente el cambio esperado en el número de Ubes es de 5 unidades menos en los sujetos disciplinados respecto a los no disciplinados ( $b=-0.474, p=0.004)$. Los que señalan tener dificultades para conocer gente nueva consumen un $69 \%$ más, es decir el valor esperado es 5.7 Ubes más a la semana respecto a los que señalan no tener dificultades para conocer gente nueva $(b=-0.527, p=0.022)$. Los sujetos que dicen tener problemas para relacionarse con las personas del sexo opuesto consumen un $45 \%$ menos, 4 Ubes menos a la semana, que los que dicen no tener problemas de relación con personas del sexo opuesto $(b=-0.592$, $p=0.006)$.

Hay una tendencia a la significación $(p=0.059)$ en aquellos que manifiestan tener problemas para decir "no", que origina una disminución del $20 \%$ en el consumo con una bajada esperada de 1.8 Ubes en el consumo semanal.

En tabaco, los sujetos que señalan tener dificultad para expresar sus sentimientos consumen un $42 \%$ más, concretamente presentan un valor esperado de 32.1 cigarrillos más a la semana que los que no presentan dificultades de expresión de sentimientos $(b=0.351, p=0.003)$. Los sujetos que dicen no tener habilidades conversacionales consumen un 56\% menos (47.7 cigarrillos menos a la semana) que los que presentan esta habilidad $(b=-0.820, p=0.001)$. Por otro lado los sujetos que señalan problemas para relacionarse con el sexo opuesto consumen 4 veces más que los que no señalan dicho problema $(b=1.398$, $\mathrm{p}<0.001)$.

Hay una tendencia a la significación $(p=0.07)$ en la estrategia de no llevar dinero, los cuales multiplican por dos la prevalencia de consumo de tabaco.

En cannabis, los sujetos que señalan saber relajarse sin drogas consumen un 78\% menos (18.2 cigarrillos de THC menos a la semana) que los que señalan necesitar las drogas para relajarse $(p=0.057)$. Los que dicen tener problemas para relacionarse con las personas del sexo opuesto consumen un $70 \%$ menos respecto a los que no presentan problemas de relación con el sexo opuesto $(b=-1.194, p=0.006)$. Se espera una disminución de 18.2 cigarrillos de THC en los sujetos que señalan saber relajarse sin drogas y una disminución de 4 cigarrillos de THC en aquellos que señalan tener 
problemas de relación son el sexo opuesto. También en esta sustancia aparece una variable con tendencia a la significación ( $p=0.057)$, que muestra que el uso de la estrategia de afrontamiento "pensar en las consecuencias negativas del consumo" disminuye el consumo en un $40 \%$, traduciéndose esto en un valor esperado de 2.5 cigarrillos de THC menos en los que piensan en las consecuencias negativas del consumo. Una segunda variable con tendencia a la significación $(p=0.061)$ corresponde a la habilidad propia de sentirse una persona disciplinada, lo que disminuye el consumo en un 33\%, con un número esperado de 2.7 cigarrillos de THC menos que los que no poseen dicha habilidad.

Cada uno de los resultados expuestos se interpretan siempre suponiendo que se mantienen constante las otras variables del modelo.

\section{DISCUSIÓN}

En nuestra muestra hemos encontrado, en la misma dirección que Barkin y otros (2002), que en bebidas fermentadas las estrategias efectivas son las referentes a habilidades asertivas en situaciones concretas de consumo. La estrategia de afrontamiento de control de estímulos "no llevar dinero" no es efectiva, posiblemente por el bajo coste de estas bebidas, mientras que si parece funcionar en nuestra muestra, tal como señalan Prochaska y DiClemente (1982, 1984), la estrategia de control de estímulos “Evitar personas y lugares relacionados con las drogas". Por otro lado la estrategia cognitiva "Pensar en las consecuencias negativas" disminuye el consumo de alcohol justificándose así el uso que se hace de esta estrategia dentro de la terapia cognitiva de las drogodependencias (Beck, Wright, Newman y Liese, 1993).

Como también encontraron Todd, Kashdan, Charlene, Vetter y Collins (en prensa), entre otros, la autodisciplina está relacionada con un menor uso de alcohol.

En la Teoría de la Activación, Eysenck (1973, 1980 y 1981) habla del uso del tabaco como reductor de sentimientos de ansiedad e ira en determinadas situaciones tensas para el sujeto. En la muestra analizada se ha obtenido que la expresión de sentimientos, y los problemas de relación con el sexo opuesto influyen positivamente en el uso de tabaco, aumentando el consumo. Esto podría apoyar la teoría de Eysenck, ya que estos déficits en habilidades sociales podrían ser causantes de estrés y por tanto la utilización de tabaco podría servir para eliminar los sentimientos de ansiedad y/o hacer frente a estas situaciones sociales.

Por otro lado, en la muestra se ha encontrado que los sujetos con déficits en habilidades conversacio- nales consumen menos tabaco. Esto podría estar relacionado con los resultados del estudio de Eysenck (1980) que hallaron que los fumadores frente a los no fumadores eran más extravertidos y uno de los aspectos que recogían en el constructo de extraversión era la sociabilidad. Un amplio estudio sobre las variables de personalidad en diferentes usuarios de tabaco y derivados puede verse en Spielberger, Reheiser, Foreyt, Poston y Volding (2004).

Podemos concluir, no obstante, que déficits en la expresión de sentimientos y en las relaciones con el sexo opuesto, pueden ser variables de mantenimiento de un mayor uso de tabaco y déficits en habilidades sociales conversacionales parece disminuir el consumo de tabaco, según los resultados obtenidos.

El consumo de cannabis aumenta en los sujetos que utilizan esta sustancia para relajarse, esto puede estar relacionado con el efecto relajante que se produce tras el consumo de cannabis (Leza y Lorenzo, 2000). El tener problemas para relacionarse con el sexo opuesto, no obstante, parece disminuir el consumo de esta sustancia.

Según estos resultados los consumidores de THC tienen menos problemas de interrelación con el sexo opuesto, por lo que puede ser posible que el consumo de THC se utilice para facilitar las relaciones con el sexo opuesto.

Como conclusión podemos señalar que las estrategias de afrontamiento generales están mayoritariamente relacionadas con el consumo de tabaco, cannabis y bebidas destiladas y únicamente en bebidas fermentadas parecen influir las estrategias de afrontamiento especificas al consumo. Así, características de los sujetos no directamente relacionadas con el consumo de drogas parecen estar influyendo en el consumo que los sujetos hacen de las bebidas destiladas, tabaco y cannabis, mientras que déficits en estrategias de afrontamiento especificas a la situación de consumo son los que llevarán a un sujeto a consumir bebidas fermentadas.

Otros estudios han encontrado diferencias en función del sexo, estatus, etc. Estos elementos, claramente, son inmutables y poco se puede hacer para modificarlos. No obstante, nuestra investigación si que indica cómo elementos alterables, como las habilidades sociales o determinadas estrategias de afrontamiento, parecen marcar la diferencia en determinar el uso que se hace de las diferentes sustancias.

Por ello, los programas de tratamiento y prevención tienen que ir encaminados a incorporar el aprendizaje de estrategias de afrontamiento generales y específicas, no solo teniendo en cuenta las variables individuales y contextuales de los sujetos, sino también el tipo de sustancia. 


\section{REFERENCIAS}

Bandura, A. (1977): Social learning theory. Englewood Cliff, N.J: Prentice-Hall.

Barkin, S.L, Smith, K.S y DuRant, R.H. (2002). Social skills and attitudes associated with substance use behaviors among young adolescents. Journal of Adolescent Health, 30. 448-454

Beck, A., Wright, F., Newman, C. y Liese, B. (1993). Cognitive Therapy of substance abuse. Guildford Press. New York.

Cameron, A. C. y Trivedi, P. K. (1998). Regression analysis of count data. Cambridge University Press.

Chaney, E.F., O'Leary, M.R. y Marlatt, G.A. (1978). Skill training with alcoholics. Journal of Consulting and Clinical Psychology, 46, 1092-1104.

Chaney, E.F., Roszell, D.K. y Cummings, C. (1982). Relapsed in opiate addicts: A behavioral analysis. Addictive Behaviors, 7, 291-297.

D'Zurilla, T.J. y Goldfried, M.R. (1971). Problem solving and behavior modification. Journal of Abnormal Psychology, 78, 107-126.

Eysenck, H.J. (1973). Personality and the maintenance of the smoking habit. In W. L. Bunn (Ed.), Smoking behavior: motives and incentives. Washington, DC: Winston/Wiley.

Eysenck, H.J. (1980). The causes and efects of smoking. London: Temple Smith.

Eysenck, H.J. (1981). A model for personality. New York: Springer.

Finney, J.W. y Moos, R.H. (1995). Entering treatment for alcohol abuse: A stress and coping model. Addiction, 90, 1223-1240.

Flowers, J.V. (1975). Simulation and role playing methods. In F. H. Kanfer y A. P. Goldstein (Eds.), Helping people change: A textbook of methods. New York: Pergamon.

Lazarus, R.S. y Folkman, S. (1984). Coping and adaptation. In W. D. Gentry (Ed.). Handbook of behavioral medicine. New York: Guilford.

Leza, J.C. y Lorenzo, P.(2000). Efectos farmacológicos de los Cannabinoides. Adicciones, vol.12, sup.2, 109-133.

Marlatt, G.A. (1985). Relapse prevention: Theorical rationale and overview of the model. En G.A. Marlatt y J.R. Gordon (eds), Relapse prevention: Maintenance strategies in the treatment of addictive behaviors. New York: Guilford

Marlatt, G.A. y Gordon, J.R. (1980). Determinants of relapse: Implications for the maintenance of behavior change.
En P.O. Davidson y S.M. Davidson (eds.), Behavioral medicine: Changing health life styles, Nueva York, Brunner/Mazel.

Marlatt, G.A. y Gordon, J.R. (1985). Relapse prevention: Maintenance strategies in the treatment of addictive behaviors. New York: Guilford

Monti, P.M., Abrams, D.B., Binkoff, J.A. y Zwick, W.R. (1986). Social skills training and substance abuse. En C.R. Hollin y P. Tower (eds.), Handbook of socials skills training, Nueva York, Pergamon Press.

Prochaska, J. y Prochaska, J.M. (1993). Modelo transteorético de cambio para conductas adictivas. En Casas y M. Gossop (eds.), Recaída y prevención de recaídas. Tratamientos Psicológicos en Drogodependencias, Barcelona, Ed. Neurociencias, Citrán.FISP.

Prochaska, J.O. y DiClemente, C.C. (1982). Transtheoretical therapy: Toward a more integrative model of change. Psychotherapy: Theory, Research and Practice, 19, 276288.

Prochaska, J. O. y DiClemente, C. C. (1984). Stages and processes of self-change of smoking: Toward and integrative model of change. Journal of Consulting and Clinical Psychology, 51, 390-395.

Prochaska, J.O., DiClemente, C.C. y Norcross, J.C. (1992). In search of how people change. Applications to Addictive Behaviors. American Psychologist, 47, 1102-1114

Spielberger, C.D., Reheiser, E.C., Foreyt, J.P., Poston, W.S.C. y Volding, D.C. (2004). Personality determinants of the use of tobacco products. Personality and Individual Differences, 36, 1073-1082.

Todd, B., Kashdan, Charlene, J., Vetter, R. y Collins, L. (2004). Substance use in young adults:Associations with personality and gender. Addictive Behaviors (en prensa).

Trower, P., Yardley, K., Briant, G.M. y Shaw, P. (1978). The treatment of social failure. Behavior Modification, 2, 41-60.

Wagner, E.F., Myers, M.G. y Ininch, J.L. (1999). Stress-coping and temptation-coping as predictors of adolescent substance use. Addictive Behaviors, 24 (6), 769-779,

Wills, T.A. y Hirky, A.E. (1996). Coping and substance abuse: A theoretical model and review of the evidence. In $\mathrm{M}$. Zeichnec \& N. S. Eudler (Eds.), Handbook of coping: Theory Research and Applications, 279-302. New York: Wiley.

Wills, T.A. y Shiffman, S. (1985). Coping and substance use: A conceptual framework. In S. Shiffman \& T. A. Wills (Eds.), Coping and Substance Use, 3-24. San Diego, CA: Academic Press. 